\title{
Knowledge-Based Design Space Exploration of Wireless Sensor Networks
}

\author{
Paolo Roberto Grassi ${ }^{1}$, Ivan Beretta ${ }^{2}$, Vincenzo Rana ${ }^{1,2}$, \\ David Atienza ${ }^{2}$ and Donatella Sciuto ${ }^{1}$ \\ ${ }^{1}$ Politecnico di Milano, DEI, Italy, \{grassi, rana, sciuto\}@elet.polimi.it \\ ${ }^{2}$ École Polytechnique Fédérale de Lausanne, ESL, Switzerland, \{ivan.beretta, david.atienza\}@epfl.ch
}

\begin{abstract}
The complexity of Wireless Sensor Networks (WSNs) has been constantly increasing over the last decade, and the necessity of efficient CAD tools has been growing accordingly. In fact, the size of the design space of a WSN has become large, and an exploration conducted by using semi-random algorithms (such as the popular genetic or simulated annealing algorithms) requires an unacceptable amount of time to converge due to the high number of parameters involved. To address this issue, in this paper we introduce a knowledgebased design space exploration algorithm for the WSN domain, which is based on a discrete-space Markov decision process (MDP). In order to enhance the performance of the proposed algorithm and to increase its scalability, we tailor the classical MDP approach to the specific aspects that characterize the WSN domain. We exploit domain-specific knowledge to choose the best node-level configuration in WSNs using slotted star topology in order to reduce the exploration time. The proposed approach has been tested on IEEE 802.15.4 star networks with various configurations of the number of nodes and their packet rates. Experimental results show that the proposed algorithm reduces the number of simulations required to converge, with respect to state-of-the-art algorithms (e.g., NSGA-II, PMA and MOSA), from 60 to $87 \%$.
\end{abstract}

\section{Categories and Subject Descriptors}

C.4 [Computer Systems Organization]: Performance of Systems-Design studies, modeling techniques; C.3 [Computer Systems Organization]: Special-Purpose and Application-Based Systems-Real-time and embedded systems

\section{General Terms}

Design, Algorithms

\section{Keywords}

Design Space Exploration, Markov Decision Process, Wireless Sensor Networks

Permission to make digital or hard copies of all or part of this work for personal or classroom use is granted without fee provided that copies are not made or distributed for profit or commercial advantage and that copies bear this notice and the full citation on the first page. To copy otherwise, to republish, to post on servers or to redistribute to lists, requires prior specific permission and/or a fee.

CODES+ISSS'12, October 7Ü12, 2012, Tampere, Finland.

Copyright 2012 ACM 978-1-4503-1426-8/12/09 ...\$15.00.

\section{INTRODUCTION}

The increasing complexity of Wireless Sensor Networks (WSNs) is leading towards the deployment of very sophisticated and ubiquitous networked systems. A WSN is composed by several nodes that communicate through a wireless channel: these nodes are typically battery-powered, and equipped with low-performance processors and small memories in order to reduce the power requirements. A common WSN node comprises five main components [19]: a processing element (microcontroller, processor, etc.), memory elements (RAM, SD, Flash, etc.), multiple communication layers (physical radio, MAC, Routing), a power supply and a sensing unit (including sensor, transducer, ADC). During the design phase, the contribution of all these components must be combined to identify the configuration that best fits the design objectives. The combination of different layers and the large number of configurable hardware and software parameters often generates an extremely large design space, which requires a powerful CAD algorithm to carry out the exploration.

A critical aspect of the Design Space Exploration (DSE) is the evaluation of a WSN configuration. Among the possible evaluation techniques, network models provide a deep understanding of the network dynamics that can be exploited during the DSE, but their definition is generally difficult and requires a deep knowledge of the working domain [4]. As a consequence, evaluation is typically performed using extensive network simulations [7], which are more accurate and reusable. However, a WSN simulation may take from several minutes to hours (depending on the network size) to be completed, and it should be repeated tens of times (generally more than 30) to mitigate the effects of randomness and ensure reliable statistical results.

In this paper, we propose a knowledge-based DSE algorithm that effectively tackles the design complexity of WSNs, by combining the domain knowledge of the analytical models with the generality and the flexibility of network simulators. Unlike semi-random algorithms, which require an evaluation at each step, the proposed approach performs several moves by relying on a set of domain-specific rules, thus greatly reducing the number of simulations. For this purpose, we adapt the Markov decision process (MDP) algorithm [5] to the WSN domain, and we solve its inherited scalability issues to face the large design spaces of WSNs. We also introduce a general domain knowledge for the most popular WSN MAC layer, i.e., the IEEE 802.15.4 [3], which can be reused on any WSN based on this protocol. Validation results prove that the proposed approach effectively 
reduces the number of simulations with respect to state-ofthe-art semi-random algorithms from 60 to $87 \%$ on multiple real-world scenarios.

\section{PROBLEM DEFINITION}

In this section, we provide a characterization of the design space (denoted as $S$ ) of WSNs, in order to show the complexity of this kind of systems. The parameter space of a WSN is divided into two parts: a set of node parameters $\left(P_{n}\right)$, which can assume different values on each nodes, and a set of network parameters $\left(P_{r}\right)$, which assume one value throughout the whole network (or at least a part of it). For example, the memory size or the type of processor are specific of each node, hence they belong to $P_{n}$, whereas the network protocol must be the same among the nodes, therefore it belongs to $P_{r}$. Each parameter $p \in\left(P_{n} \cup P_{r}\right)$ is assigned to a discrete set of values in an interval $\left[p_{\min }, p_{\max }\right]$, whose cardinality is denoted as $|p|$.

Node parameters heavily affect the design space size $|S|$, since each parameters can assume an independent value on each node. Thus, when network size (expressed in terms of the number of nodes, $N$ ) increases, then $|S|$ increases exponentially. More formally, we can express the size of the design space of a WSN:

$$
|S|=\left(\prod_{p \in P_{r}}|p|\right) \cdot\left(\prod_{q \in P_{n}}|q|\right)^{N} .
$$

To understand the order of magnitude of $|S|$, let us show a relatively small example for structural or environmental monitoring. Let us assume that 8 nodes are placed on a $2 \times 2 \times 2$ tridimensional grid in the monitored area, and that the communication is regulated by the IEEE 802.15.4 MAC protocol [3]. Even without considering any hardware parameter, the configuration of the MAC protocol itself contains a wide set of possible parameters in $P_{r}$ (e.g., the frame and the beacon orders [3]) and in $P_{n}$ (e.g., the choice of using the contention active communication, or the number of requested guaranteed time slots [3]). Overall, the design space contains approximately 290 billions of solutions.

Thus, an extensive DSE would take an unacceptable amount of time when network simulation is the only viable way to evaluate a solution. As a consequence, a technique that is able to reduce the number of simulations is required.

\section{STATE OF THE ART}

Design space exploration is a well established research topic in many different fields, such as embedded architectures or system-level design. However, automatic DSE is still immature in WSN design, where the complexity of the network and the peculiarity of each application domain often push the developers towards manual and ad hoc optimizations (e.g., the work in [10] for wildlife monitoring).

The main obstacle towards the complete automation of the DSE of WSNs is a rapid and accurate evaluation of a solution. Currently, the two evaluation techniques [4] are models and simulations. Analytical models describe the WSN dynamics by means of a set of equations that provide a white-box view of the system, thus extensive analysis are possible. When a model-based evaluation is employed, the DSE is considerably faster compared to simulation-based
Table 1: Speed and accuracy of existing DSE techniques

\begin{tabular}{c|c|c}
\hline & Low Accuracy & High Accuracy \\
\hline Slow & & $\begin{array}{c}\text { Simulation-based } \\
([7][16][13])\end{array}$ \\
\hline Fast & $\begin{array}{c}\text { Model-based } \\
([8][15])\end{array}$ & MDP \\
\hline
\end{tabular}

approaches, but the accuracy of the model is typically guaranteed only for specific domains or for specific aspects of the WSN. In fact, only models that have been proposed for single classes of WSNs (e.g., [21] for body area networks) or for specific protocols (e.g. [11],[6] and [18] for three different operations of the IEEE 802.15.4 MAC layer) show an accuracy that is comparable to a simulation. In [14] an MDP is used to detect optimal sensor node operation in order to meet the application requirements. In this case, the solution is identified analytically solving the MDP via custom reward functions. However, it requires an accurate model to both guide and evaluate the solutions, which is a very important limitation. On the other hand, simulations better profile all the aspects related to communication, energy and resource usage of distributed applications. However, a robust simulation takes several from minutes to hours, thus making it impractical for extensive DSEs.

In this context, the evaluation technique heavily affects the choice of the optimization algorithms. When an analytical description of the WSN (or, at least, of a specific part of it) is available, the optimization can be performed using ad hoc heuristic algorithms (e.g., in [8] to solve the network connectivity problem) or efficient techniques such as convex optimization (e.g., [15] for energy/delay optimization). The simulation-based estimation, on the other hand, has a black-box nature that does not allow any analytical consideration during the execution of the algorithm. This limitation leads to the employment of semi-random approaches such as genetic algorithms (e.g., [7] for placement and role assignment), multi-objective evolutionary algorithms (e.g., [16] for gossip-based WSNs), simulated annealing and Bayesian networks (e.g., [13] for a cross-layer optimization of cognitive wireless networks). However, given the high execution time required by a simulation, the class of semi-random algorithms does not scale well on large design spaces that are typical of real-world WSNs.

According to these considerations, model-based and simulation-based exploration are currently the two alternative approaches for the DSE of WSNs, and each one offers a speed/accuracy tradeoff that is summarized in Table 1. In this work, we aim at combining the high accuracy and reliability of a solution that is evaluated by a simulator, and the high speed that can be achieved when we include model information, calibrated with the simulator, in the DSE. In particular, the rationale is to move within the design space by exploiting the model information until they are accurate, and then use the simulation whenever it is strictly necessary.

\section{MARKOV DECISION PROCESS}

In this work we propose a knowledge-based design space exploration algorithm for the WSN domain, which is based on a discrete-space Markov decision process (MDP). In particular, we tailor the the classical MDP approach, which has been successfully applied in other domains such as multipro- 
cessor systems design [5], to the WSN domain in order to enhance its performance and to increase its scalability. The proposed algorithm combines the available domain knowledge - which may come from an analytical model or by an analysis of the specific application - with a simulation-based network evaluation, in order to obtain an accurate and yet efficient DSE for WSNs.

In our approach, the DSE is considered as a path from the initial configuration $P$ to a final configuration $\widehat{P}$. The path is identified by applying sequential transformations on the parameters, and the quality of these transformations is evaluated thanks to both models and simulations. The configuration $P$ is composed by a set of parameters such as $P=\left\{p_{1}, \ldots p_{k} \ldots p_{n}\right\}$ and an action $a \in A$ specifies how a configuration should be modified (i.e. "double the CPU frequency"). Actions transforms the configurations as follows:

DeFinition 1. Given a configuration of parameters $P=$ $\left\{p_{1}, \ldots p_{k} \ldots p_{n}\right\}$ and an action $a \in A$, a transformation $\tau\left(p_{k}, a\right)$ produces a new configuration $P^{\prime}=\left\{p_{1}, \ldots p_{k}^{\prime} \ldots p_{n}\right\}$ where $p_{k} \neq p_{k}^{\prime}$

Once a transformation $\tau(p, a)$ has been performed, its effect on the metrics is evaluated using movement vectors:

Definition 2. A movement vector is a vector of intervals in the metrics space corresponding to a transformation vector in the parameter space defined as:

$$
\Phi=\left\langle f_{1}\left(\tau\left(p_{k}, a\right)\right), f_{2}\left(\tau\left(p_{k}, a\right)\right), \ldots f_{i}\left(\tau\left(p_{k}, a\right)\right),\right\rangle
$$

where $i=|M|$, and $\vec{f}=f_{1}, f_{2}, \ldots, f_{i}$ are functions that determine the effect of the transformation $\tau$ on each metric $m_{j} \in M$.

Movement vectors are used to estimate the metrics of a configuration $P^{\prime}$ generated from an action $a$ applied to a configuration $P$. For each metric $m_{i} \in M$, an interval in the metrics space $\left[m_{i}^{L}, m_{i}^{H}\right]$ specifies the range where the actual (real) value of the metric is included. $\vec{f}$ functions and movement vectors $\Phi$ are problem-specific (based on a model), thus problem-specific models are required.

For instance, the effect of the action "double the operational frequency of a processor" could in the worst case increase of up to two times of the energy consumption $\left(\mathrm{m}^{H}\right)$ or, in the best case, leave it unaltered $\left(m^{L}\right)$. Thus, the movement vector associated with this action is $[E, 2 E]$, where $E$ indicates the current energy consumption. It indicates that, whereas the action "double the operational frequency of a processor" is applied on a configuration $P$, the energy consumption of the resulting configuration $P^{\prime}$ belongs to the interval $[E, 2 E]$. In Section 5 , we provide a set of movement vectors for networks based on the IEEE 802.15.4 protocol.

To tackle exploration accuracy, the metrics space is partitioned according to a parameter $\vec{\lambda}$ defined for the exploration. $\vec{\lambda}$ is a vector of scalars that specifies the maximum width of a partition for each metric. Partitioning is used to divide large areas into smaller areas such as the maximum error is defined by $\lambda$, given by the difference between the present value of the metrics (to be determined through simulation) and the centroid of the partition that best approximates it. An example of an action on a parameter is illustrated in Figure 1 where action $a$, applied to $P$, generates $P^{\prime}=\tau(P, a)$. The metrics $M\left(P^{\prime}\right)$ are evaluated from

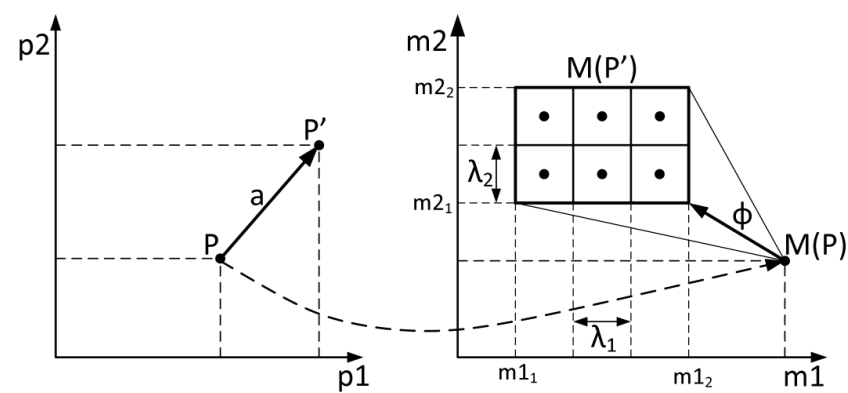

Figure 1: An example of actions on P. The metrics space is partitioned in 6 areas and centroids are illustrated with black dots

$M(P)$ through domain knowledge $\Phi$ and results in an area included into $\left\langle\left[m 1_{1}, m 1_{2}\right],\left[m 2_{1}, m 2_{2}\right]\right\rangle$. The area is partitioned in six partition (whose centroids are depicted with black dots) according to $\vec{\lambda}$.

The utility function $(\Psi)$ has the utility property [20] and is a function of the metrics $M$. In a multi-objective exploration, metrics are combined in order to be able to enumerate the solutions. $\Psi$ can be linear $\left(w_{1} m_{1}+w_{2} m_{2}+\ldots+w_{i} m_{i}\right)$ or exponential $\left(m_{1}^{w_{1}}+m_{2}^{w_{2}}+\ldots+m_{i}^{w_{i}}\right)$. To obtain the pareto curve, the exploration is performed in a multivariate environment, thus $\vec{W}=\left\{w_{1}, w_{2}, \ldots w_{i}\right\}$ must change during the exploration in order to explore all the directions of the metrics space. In a two-dimensional metrics space, this can be achieved, i.e., using the scalarizing function $m_{1}^{\alpha} m_{2}^{(1-\alpha)}$ with $\alpha$ in $[0,1]$.

The exploration is modeled as a MDP:

Definition 3. A MDP is a tuple $<S, A, T P, R>$ where:

- $S$ is the set of possible states describing a solution of the DSE problem;

- $A$ is the set of possible actions that can be applied on the states;

- $T P: S \times A \rightarrow \Pi(S)$ is the state transition function as the probability density function for every state-action pair;

- $R: S \times A \times S^{\prime} \rightarrow R$ is the expected reward for each state-action pair.

The behavior of the MDP is described with the Decision Tree $D$, a tree-based structure used to evaluate the various configurations. A node $s \in S$ in $D$ represents a state of the system expressed by the tuple $\langle P, M\rangle$, where $P$ is a point in the parameters space and $M$ a point in the metrics space. An edge $e \in E$ is defined as:

$$
e=\left\langle s_{i}, s_{j}, a, \operatorname{TP}\left(s_{i}, a, s_{j}\right)\right\rangle
$$

and it represents the transition probability (given by the transition function $T P$ ) from state $s_{i}$ to state $s_{j}$ when action $a$ is applied.

Each partition identifies a new node in the decision tree, and the reward of the actions $(R)$ is computed as the difference between parent $\left(\Psi_{p}\right)$ and child $\left(\Psi_{c}\right)$ utility functions:

$$
R=\Psi_{p}-\Psi_{c}
$$

For each partition $\left\langle s, a, s^{\prime}\right\rangle$, the probability $T P_{i}\left(s_{i}, a, s_{k}\right)$ is computed as the number of times $\langle s, a\rangle$ ends in $s^{\prime}$ when traversing the decision tree from root to leaf. The decision 


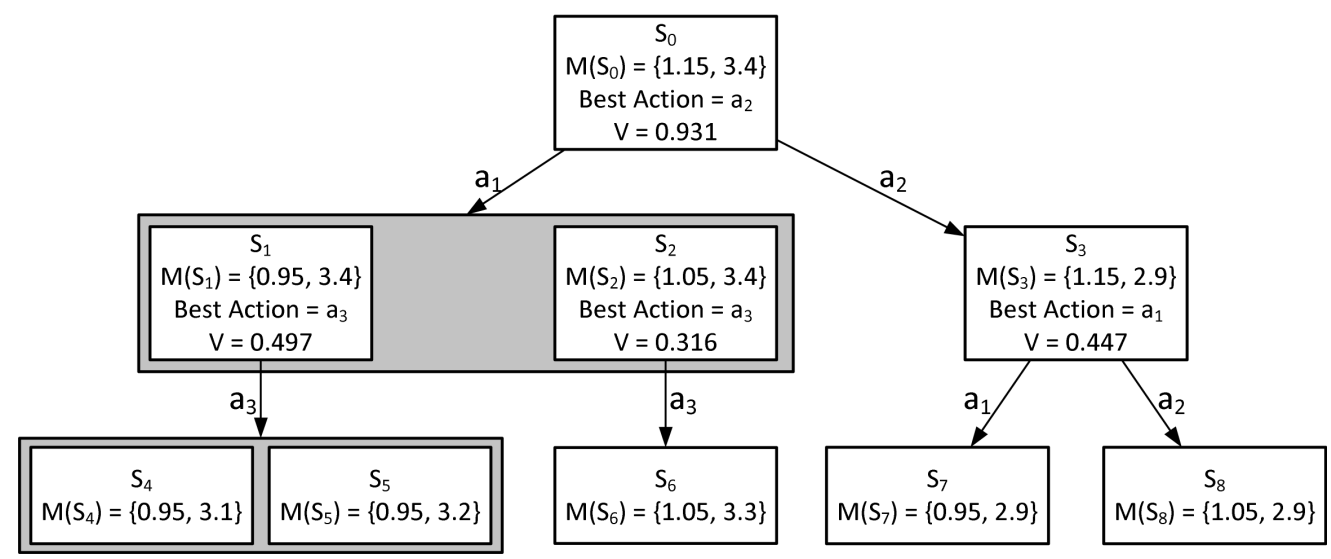

Figure 2: Example of a Decision Tree $D$. Cumulative return $V$ is computed using the Value Iteration Algorithm (see Algorithm 1)

tree is progressively built by iterating on the newly generated nodes breadth-first, until either is not possible to apply any other action on the leaf nodes or the $l$-th level is reached. During the creation of $D$, in order to avoid useless exploration, opposite actions are included into a forbidden list such as they will not be applied. For example, if the action Increase CPU frequency is applied, the action Decrease $C P U$ frequency in included into the forbidden list since it voids the previous action.

An example of the Decision Tree $D$ is shown in Figure 2. From the initial state $s_{0}$, two actions are applied $\left(a_{1}, a_{2}\right)$ resulting in three states $\left(s_{1}, s_{2}, s_{3}\right)$ where $s_{1}$ and $s_{2}$ are two partitions. Actions $a_{3}$ and $a_{1}$ are further applied on the second level of the tree. For each state, metrics are associated; this example has two metrics that must be minimized. Rewards estimate the benefits of the actions on $\Psi$ and are used to guide the exploration to better solutions. To determine the reward of the actions, Value Iteration Algorithm (see Algorithm 1) is used on $D$. For all the states in the tree and for all the available actions, the reward of an action $Q(s, a)$ is computed by adding the reward of choosing that action $\left(R\left(s, a, s^{\prime}\right)\right)$ with the cumulative return on the destination node $\left(V\left(s^{\prime}\right)\right)$ scaled by $\gamma \cdot \gamma$ is a scalar value $(0 \leq \gamma \leq 1)$ used to control the influence of (expected) cumulative returns. Transition probability $T P\left(s, a, s^{\prime}\right)$ is considered in the formula. In this specific example, $V$ is computed by considering $\gamma=0.6$ and the utility function $m_{0}^{1-\alpha}+m_{1}^{\alpha}$ with $\alpha=0.5$. According to the metrics, the algorithm identifies $a_{2}$ as the best action in this situation; in fact, $s_{7}$ is optimal with respect to the given metrics. The new state is $s_{3}$ and no simulation is required since no uncertainty is detected here.

The overall exploration strategy is illustrated in Algorithm 2. It starts (first step) by generating an initial configuration $P_{0}$ (line 5) - that can be identified randomly or pseudo-randomly. Once $P_{0}$ has been evaluated by simulation and the initial state $s_{0}$ has been generated (line 6), the set of states to be examined $(\bar{S})$ is initialized (line 7 ).

The second step of the algorithm solves the MDP. For each state in $\bar{s}$, all possible actions are applied, generating the configurations that differ from $s_{0}$ by one parameter (lines 13-26). For all the generated configurations (obtained by applying $a$ in $\left.s_{i}\right), s_{k}$ metrics are partitioned, $D$ and $\operatorname{TP}\left(s_{i}, a, s_{k}\right)$ are updated. The generation of the Deci-

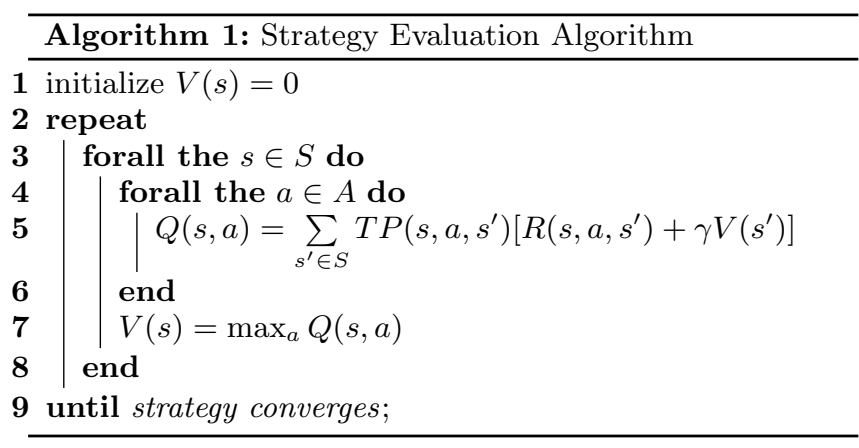

sion Tree continues until no new states are available or the maximum depth $l$ has been reached. At this point (line 27) the best value iteration algorithm [20] is applied and the best_actions are updated on $D$.

The third step applies the best_action of $s_{0}$ in $s_{0}$ to get the set of reachable states $(N S)$. At this point, three situations are possible:

1. No Uncertainty: the action can lead to a single state. The action is determined with an accuracy $\lambda$ and no simulation is necessary;

2. Uncertainty of the First Kind: the action leads to a set of states and the algorithm maps the same action to all of them. It means that, whichever state the system will end into, the same next action is chosen. In case the amount of states is below a given threshold $K$, simulation is not required and parallel exploration will follow, otherwise simulation is required; $\mathrm{K}$ controls the amount of solutions to be explored and is used to tackle the scalability of the algorithm. Parallel explorations could evolve differently since they start from different partitions, thus they must be considered separately;

3. Uncertainty of the Second Kind: the action leads to a set of states, but the algorithm maps two or more different actions on those states. In this situation, simulation is needed to determine to which state the action really leads to.

Three examples of uncertainties are depicted in Figure 3. In $3(\mathrm{a})$ an uncertainty of the first kind is detected and $|N S|<$ $K$ thus simulation is not required. In $3(\mathrm{~b})$ an uncertainty of 


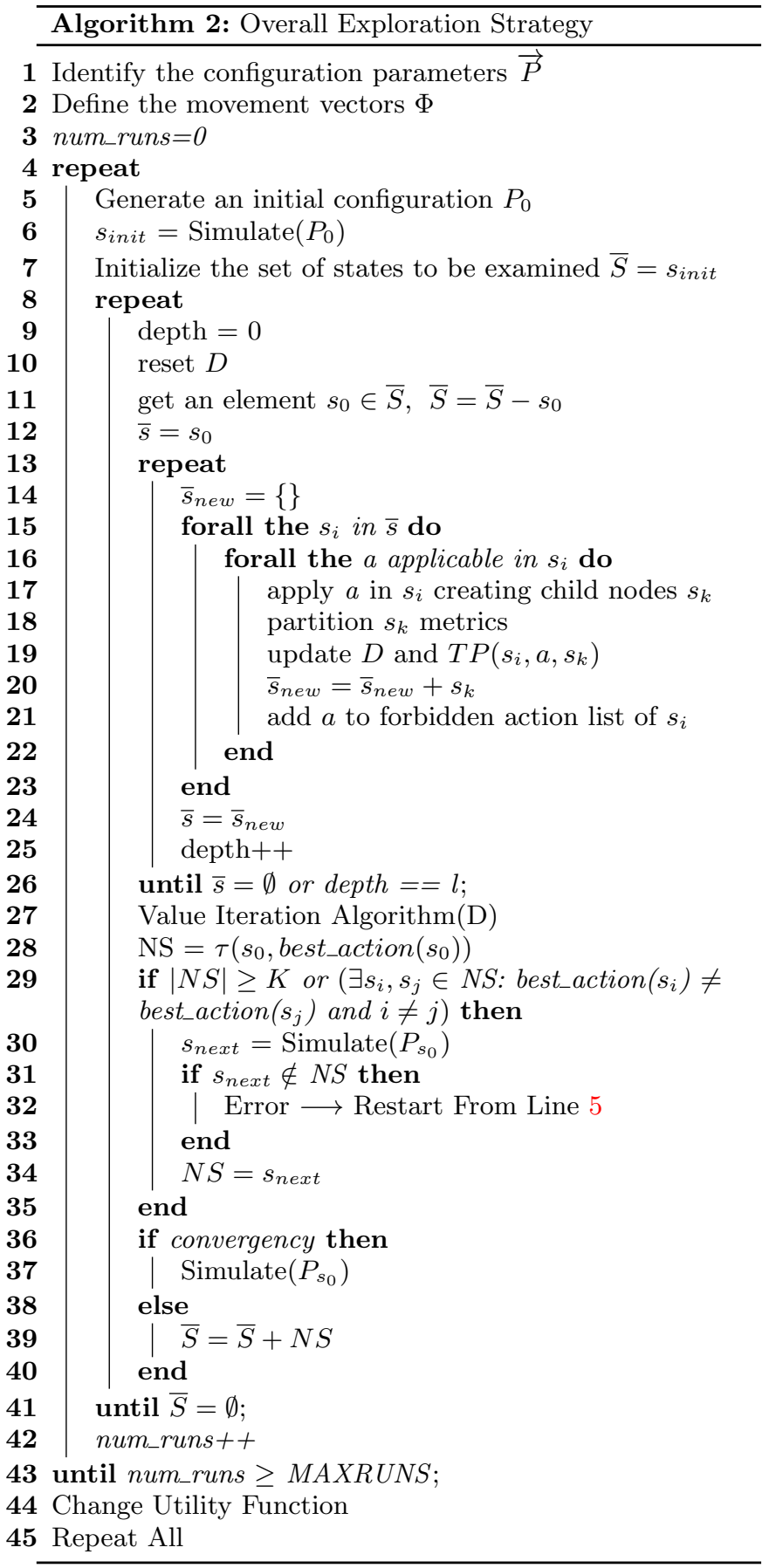

the first kind is detected but $|N S| \geq K$ thus simulation is required. In $3(\mathrm{c})$ simulation is required since an uncertainty of the second kind is detected.

In other terms, simulation is performed only if the cardinality of $N S$ (the amount of states reached by the best action) is above a given value $K$ or the action $a$ brings the system to a set of states and the Value Iteration Algorithm mapped different actions to each of those states. In case simulation results $s_{\text {next }}$ are not contained in $N S$ exploration restarts from line 5 , otherwise $N S$ is update with the real value $s_{\text {next }}$. At this point the algorithm checks if convergency has been reached (line 36); in the positive case, a

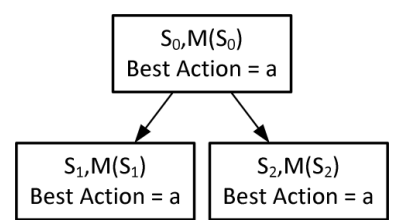

(a) Simulation not required

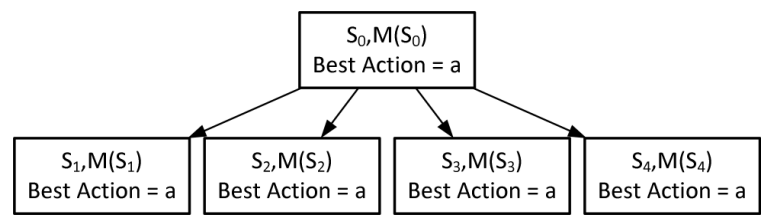

(b) Simulation required

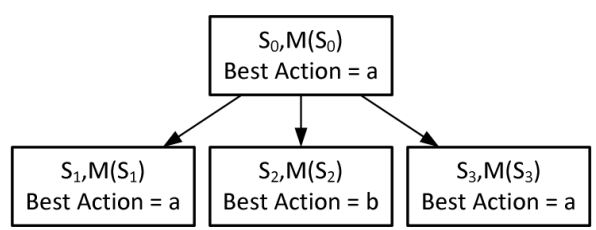

(c) Simulation required

Figure 3: Uncertainties of First and Second Kind with $K=3$

simulation is performed (line 37) to get the real values of the metric of $s_{0}$ (unless it has been previously simulated), otherwise, $\bar{S}$ is updated and the exploration continues.

Step one, two and three are repeated until a maximum number of runs (MAXRUNS) has been performed.

In the last step, the weights $\vec{w}$ of the utility function $\Psi$ are updated (line 44) and the algorithm is entirely repeated. It allows to change the direction of the exploration in order to cope multi-objective optimizations. The algorithm stops when no more utility functions would be used.

In conclusion, the algorithm explores all the possible actions for all the reachable states with an event horizon of $l$ to determine an optimal local action considering various sequences of actions. The effectiveness of the approach strictly depends on the quality of the movement vectors; as the accuracy of movement vectors increases, the amount of required simulations decreases. In fact, in the optimal case, movement vectors identify areas with size lower than $\lambda$, thus $N_{o}$ Uncertainty is detected. In this case, assuming that movement vectors are accurate, simulation is required at the beginning (line 6) and end (line 37) only, thus simulations are minimized and exploration speed is maximized. In the worst case, non accurate movement vectors may conduct to $U n$ certainties of First (with $|N S|>K$ ) or Second Kind, thus simulations are required at each step, leading to slower explorations. More generally, to control the accuracy and the speed of the exploration (number of states in the MDP) two mechanisms have been identified:

- Control the minimum desired accuracy with $\lambda$. It identifies the size of the partitions during the generation of the Decision Tree; increasing $\lambda$ reduces the number of partitions (states), improving the evaluation speed, but it increases the approximation error;

- Define a good event horizon $l$, which determines the maximum depth of the Decision Tree. It limits the number of steps required to the creation/evaluation 
of the MDP. Reducing $l$ improves the speed of MDP evaluation since fewer states are generated into the decision tree $D$. On the other hand, the higher is $l$ the higher is the lookahead of the algorithm.

\section{DOMAIN KNOWLEDGE DEFINITION FOR IEEE 802.15.4 NETWORKS}

The proposed approach requires movement vectors to guide the exploration to optimal solutions. In this section, we discuss a domain knowledge definition for the IEEE 802.15.4 MAC layer. The proposed MDP does not require accurate movement vectors to operate [5], thus it is not required that the users give accurate models to use this methodology; moreover, movement vectors are reusable. However, the analysis proposed in this Section has two main goals: first, to show in practice how to build a set of domainspecific rules to exploit the potential of the MDP algorithm. Second, it provides a good characterization of one the most popular MAC protocols in WSNs, hence the proposed rules can be reused.

The IEEE 802.15.4 standard [3] has been introduced to satisfy energy requirements of emergent devices. The protocol is quite common, thus the knowledge domain defined in this Section can be useful for future works. The results presented here are based on the models presented in [12] [21] [11] [6] [9] [18]. IEEE 802.15.4 network is composed by a central node, called coordinator, and a set of nodes, called members. In IEEE 802.15.4, the coordinator is the head of the network and determines the structure of the communication. In the standard, the communication is divided into sequential frames delimited by specific packets called beacons (Figure 4). A frame is divided into an active and an inactive period, and the active part is further divided in two periods named Contention Active Period (CAP) and Contention Free Period (CFP). During the CAP, nodes access the channel by using the CSMA/CA protocol, while, during the CFP, the nodes access the channel using a time division protocol which slots, namely Guarantee Time Slots (GTS), are assigned by the coordinator by means of a policy First Come First Served (FCFS) [3].

The metrics of interests are Average Energy Consumption (E), to be minimized, and Percentage of Packets Received (P), to be maximized. The IEEE 802.15 .4 is characterized by a certain amount of node and network parameters. For the sake of simplicity, we decide to restrict the analysis to four parameters: SuperframeOrder, BeaconOrder, enableCAP and requestGTS. According to the state of the art, these parameters have considerable effects on the metrics of interests, thus their optimization is important to the final design. In all the equations the following constraint

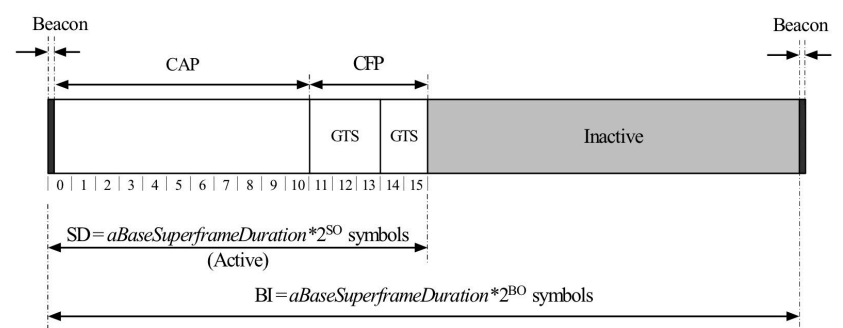

Figure 4: An example of the superframe structure (from [3]) must be satisfied:

$$
(0 \leq P \leq 100) \wedge(E \geq 0)
$$

In case the equation is not satisfied, $\mathrm{P}$ and $\mathrm{E}$ are set to the nearest value which satisfies the equation. I.e., if $P<0$ then $P$ is set to zero. The next two sections presents in detail the movement vectors. In all the equations, $E$ and $P$ represents the actual value of the metrics while $\widehat{E}$ and $\widehat{P}$ represents their estimated (next) value.

\subsection{Beacon Order and Superframe Order}

Beacon Order (BO) and Superframe Order (SO) define the main structure of the superframe since they determine the distance between the beacons and the size of the active period. The ratio between $\mathrm{SO}$ and $\mathrm{BO}$ defines the duty cycle between active and inactive period. The overall effect of increasing BO and decreasing SO is similar, since both actions modify the duty cycle in the same way. Increasing $\mathrm{BO}$ or decreasing $\mathrm{SO}$ will halve the duty cycle, thus both $\mathrm{E}$ and $\mathrm{P}$ can be reduced by 2 . Resulting movement vectors to actions increase $\mathbf{B O}$ and decrease $\mathbf{S O}$ are:

$$
\begin{aligned}
& \widehat{E}=\left[\frac{E}{2}, E\right] \\
& \widehat{P}=\left[\frac{P}{2}, P\right]
\end{aligned}
$$

On the other hand, actions that decrease BO and increase SO have an opposite behavior, since duty cycle is doubled:

$$
\begin{aligned}
\widehat{E} & =[E, 2 E] \\
\widehat{P} & =[P, 2 P]
\end{aligned}
$$

All these actions can be applied if and only if the constraint:

$$
S O \leq B O
$$

is satisfied. This constraint is imposed by the standard [3].

\subsection{Guaranteed Time Slots}

Each node requires a certain amount of GTS to the coordinator, according to the requestGTS parameter. The coordinator assigns the GTS according to the FCFS policy. It implies that if requested GTS are not designed properly, performances of the network dramatically decreases.

The maximum amount of available slots in slotted IEEE 802.15.4 is given by the following formula:

$$
M=N S S-\left\lfloor\frac{\min C A P}{B S D * 2^{F O}}\right\rfloor
$$

where NSS is the Number of Superframe Slots, minCAP is the minimum number of symbols in CAP and BSD is the Base Slot Duration.

The average amount of slots per node is equal to:

$$
A=\frac{M}{N}
$$

and the overall amount of requested slots is equal to:

$$
U=\sum_{i=0}^{N} G(i)
$$


where $G(i)$ represents the value of requestGTS of node $i$. From [18] and experimental results, we notice that, increasing the GTS requests improves $\mathrm{P}$ and reduces $\mathrm{E}$. $\mathrm{P}$ is improved because contention is reduced and $\mathrm{E}$ decreases since nodes wake-up are scheduled more efficiently in CFP with respect to CAP. In particular, when a GTS slot is allocated and few packets are in the buffer, the node sleeps during CAP and wake-ups just at the beginning of the GTS slot. In addition, since IEEE 802.15.4 uses a TDMA protocol during CFP, no additional energy is required to perform Carrier Sense.

According to the FCFS policy, if GTS have been already allocated and it is not possible to satisfy the request, the request is rejected and the node is obliged to communicate into the CAP. GTS requests are rejected if the amount of requested GTS slots (Equation 11) overcomes the maximum amount of slots in CFP (Equation 9). Moreover, in order to balance GTS requests, the amount of GTS requests per node $n(G(n))$ should not overcome the average GTS requests (Equation 10). The effect of increasing/decreasing GTS is limited to a single-slot of a single-node, so the value of $\mathrm{P}$ and E should be divided by $N M$ to provide more accurate movement vectors.

Action increase requestGTS on node $\mathrm{n}$ results in:

$$
\begin{aligned}
& \widehat{E}= \begin{cases}{\left[E-\frac{E}{N M}, E\right]} & \text { if } U \leq M \wedge G(n) \leq A \\
{\left[E, E+\frac{E}{N M}\right]} & \text { otherwise }\end{cases} \\
& \widehat{P}= \begin{cases}{\left[P, P+\frac{P}{N M}\right]} & \text { if } U \leq M \wedge G(n) \leq A \\
{\left[P-\frac{P}{N M}, P\right]} & \text { otherwise }\end{cases}
\end{aligned}
$$

On the other hand, action decrease requestGTS has the following movement vectors:

$$
\begin{aligned}
& \widehat{E}= \begin{cases}{\left[E, E+\frac{E}{N M}\right]} & \text { if } U \leq M \wedge G(n) \leq A \\
{\left[E-\frac{E}{N M}, E\right]} & \text { otherwise }\end{cases} \\
& \widehat{P}= \begin{cases}{\left[P-\frac{P}{N M}, P\right]} & \text { if } U \leq M \wedge G(n) \leq A \\
{\left[P, P+\frac{P}{N M}\right]} & \text { otherwise }\end{cases}
\end{aligned}
$$

\subsection{Enable CAP}

Considering the enableCAP parameter, two actions are possible: activate and deactivate CAP. The overall effect of enabling CAP is the increase of energy consumption (due to CAP period) and an increase of packets received. Similarly to GTS, the action has an effect on a single node only, thus both $\mathrm{E}$ and $\mathrm{P}$ changes are scaled to N. Moreover, the higher is the amount of GTS requests of a node, the lower is the effect of activation/deactivation of CAP, so the metrics are divided by $\mathrm{G}(\mathrm{n})+1$.

Differently from energy, packets received have a known behavior in case $G(n)$ is equal to zero. In fact, when $G(n)=$ 0 and CAP is not enabled, the amount of packets sent by a node $n$ is equal to zero, thus activating CAP when $G(n)=0$ has a increases the number of packets received $\left(\left[\frac{P}{N}, \frac{100}{N}\right]\right)$; the deactivation of CAP when $G(n)=0$ has the opposite effect. The movement vectors of the action activate CAP are:

$$
\widehat{E}=\left[E, E+\frac{E}{N(G(n)+1)}\right]
$$

$$
\widehat{P}= \begin{cases}{\left[P+\frac{P}{N}, P+\frac{100}{N}\right]} & \text { if } G(n)=0 \\ {\left[P, P+\frac{P}{N(G(n)+1)}\right]} & \text { otherwise }\end{cases}
$$

for the action deactivate CAP, the movement vectors are:

$$
\begin{gathered}
\widehat{E}=\left[E-\frac{E}{N(G(n)+1)}, E\right] \\
\widehat{P}= \begin{cases}{\left[P-\frac{100}{N}, P-\frac{P}{N}\right]} & \text { if } G(n)=0 \\
{\left[P-\frac{P}{N(G(n)+1)}, P\right]} & \text { otherwise }\end{cases}
\end{gathered}
$$

\subsection{Initial Points Selection}

In the classical MDP, the set of initial points is randomly generated but, especially with large design spaces, the probability to obtain bad (or even unfeasible) solutions is high. Therefore, the selection of the initial points can be guided by the model since, considering that the knowledge base has been already created to compute the actions, the same information can be used to extract the initial points.

To define the rules for the selection of the initial points, we conduct several experiments on star networks with 4,6 and 8 nodes with various packet rates $(5,15,30,50,65$, $\left.80\left[\frac{\mathrm{pkts}}{\mathrm{sec}}\right]\right)$ in order to cover a large set of applications. All the experiments where conducted with Castalia simulator [2] (see next Section). From the experimental results, we notice that assigning a request $G T S$ greater than the average (A), reduces the quality of the solution, so we suggest to create the initial population with a starting value of request$G T S$ randomly chosen in the interval $[0, A]$. In addition, a value of Beacon Order lower than 3 or greater than 12 does not usually provide good results, thus we generate the initial solution with BO included into $[3,12]$. Another aspect concerns the duty cycle $\left(\frac{S O}{B O}\right)$; good solutions usually have a duty cycle included into $[0.25,0.85]$ in all the configurations.

Summarizing, to determine the initial points, we propose to generate the set of initial points in such a way:

$$
\left\{\begin{array}{l}
0 \leq G(n) \leq A \quad \forall n \in N \\
3 \leq B O \leq 12 \\
0.25 \leq \frac{S O}{B O} \leq 0.85
\end{array} \quad \forall n\right.
$$

so that these constraints are all satisfied.

Experimental results in the next Section show that a considerable improvement on search efficiency is obtained if initial points are determined using this technique.

\section{EXPERIMENTAL RESULTS}

This Section presents two sets of experiments to validate the proposed approach. The solutions were evaluated with Castalia [2], a popular simulator for Wireless Sensor Networks and Body Area Networks, based on the OMNeT++ framework [22]. The design space for all the experiments is presented in Table 2. The design space has been explored in four different scenarios described in Table 3. These scenarios have been chosen in order to cover a large set of applications (i.e. Body Area Networks).

Since the cardinality of the design space is extremely high in all the scenarios, the optimal Pareto curve cannot be extracted with an exhaustive search, thus it has been obtained running all the exploration algorithms for 3000 iterations (solutions) for 20 iterations each. The distance between the 
Table 2: Explored Design Options for the Experimental Results

\begin{tabular}{c|c|c}
\hline Parameter & From & To \\
\hline \hline Beacon Order & 1 & 14 \\
\hline Frame Order & 1 & 14 \\
\hline Enable Cap & false & true \\
\hline requestGTS & 0 & 6 \\
\hline
\end{tabular}

Table 3: Experimental Scenarios

\begin{tabular}{l|c|c|c}
\hline & $\begin{array}{c}\text { Num of } \\
\text { Nodes }\end{array}$ & $\begin{array}{c}\text { Packet Rate } \\
\text { (Pkts/Sec) }\end{array}$ & $\begin{array}{c}\text { Size of the } \\
\text { Design Space }\end{array}$ \\
\hline \hline Scen. 1 & 4 & $30,65,80$ & $7.5 * 10^{6}$ \\
\hline Scen. 2 & 6 & $15,40,60$ & $1.4 * 10^{9}$ \\
\hline Scen. 3 & 8 & $5,15,30$ & $2.9 * 10^{11}$ \\
\hline Scen. 4 & 10 & $5,10,15$ & $5.6 * 10^{13}$ \\
\hline
\end{tabular}

Pareto sets have been compared using the Average Distance from Reference Set (ADRS) [17]. The ADRS is usually measured in terms of percentage and should be minimized.

\subsection{Evaluation of the Proposed Algorithm}

The first set of experiments aims at evaluating the improvements achieved thanks to the tailoring of the standard MDP technique to the WSN field. In particular, Table 4 presents a comparison between the standard implementation of the MDP algorithm and the one proposed within this work. The experimental results shown in Table 4 demonstrate that the ADRS of the initial points of the proposed algorithm is considerably lower than the ones obtained with [5]. This advantage makes it possible to increase the overall performance of the algorithm, so that the ADRS of the final solutions found by our algorithm is always lower than the ones obtained with [5]. In addition to this, the proposed algorithm is able to converge with a lower number of evaluations, except for Scenarios 3 and 4, where the algorithm presented in [5] often falls in local minima (as shown by the quite high ADRS of the solutions found by the algorithm).

A critical aspect of the standard MDP algorithm is its scalability. In order to analyze this factor, we suggest to handle uncertainties of the first kind with simulations only if the number of states $(|N S|)$ is larger than a given threshold K. To evaluate the effect of $\mathrm{K}$ on the exploration speed

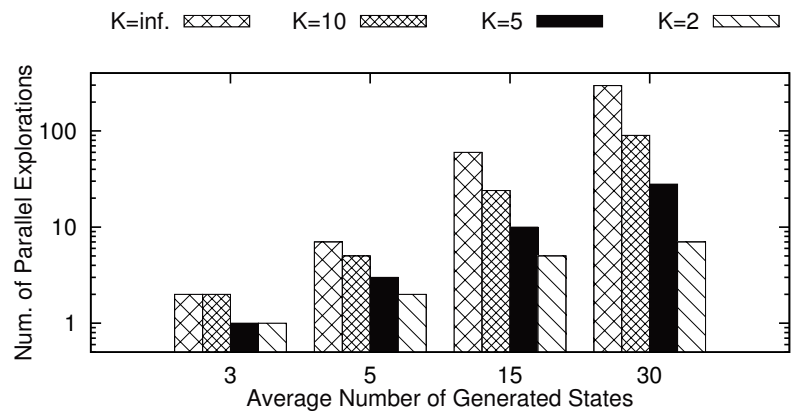

Figure 5: Number of parallel explorations with different values of $K$ ( $K=$ inf. refers to the original algorithm). The average number of generated states depends on the chosen $\lambda$ and movement vectors' accuracy
Table 4: Comparison between original and proposed implementation of the MDP

\begin{tabular}{c|c|c}
\hline Scenario 1 & {$[5]$} & [proposed work] \\
\hline ADRS[\%] init & 45.5 & 21.7 \\
ADRS[\%] final & 3.9 & 2.34 \\
Eval. for Convergence & 110 & 70 \\
\hline \hline Scenario 2 & {$[5]$} & [proposed work] \\
\hline ADRS[\%] init & 44.7 & 21.47 \\
ADRS[\%] final & 5.73 & 2.70 \\
Eval. for Convergence & 170 & 120 \\
\hline \hline Scenario 3 & {$[5]$} & [proposed work] \\
\hline ADRS[\%] init & 35.93 & 24.3 \\
ADRS[\%] final & 11.35 & 3.52 \\
Eval. for Convergence & 70 & 80 \\
\hline \hline Scenario 4 & {$[5]$} & [proposed work] \\
\hline ADRS[\%] init & 38.93 & 27.3 \\
ADRS[\%] final & 14.18 & 5.16 \\
Eval. for Convergence & 110 & 70 \\
\hline
\end{tabular}

we perform several experiments with different values of $\mathrm{K}$; Scenario 2 has been chosen as reference example for this analysis. We vary the values of $\lambda$ such as the average number of generated states for each action is between 3 (higher $\lambda$ ) and 30 (lower $\lambda$ ). Figure 5 illustrates the average number of parallel explorations for different values of $\mathrm{K}$ with respect to the average number of states for each step. The bigger is $\mathrm{K}$, the higher is the amount of parallel (independent) explorations. However, the effective exploration time is not directly correlated with the amount of parallel explorations. Figure 6 illustrates the average amount of time (in minutes) required for the exploration with different values of K. Although the number of parallel evaluations increases with both $\mathrm{K}$ and the number of generated states, the overall time behaves differently. In fact, for small values of generated states, $K=5$ performs better then $K=2$ even if the amount of parallel exploration is bigger. However, for high values of generated states (i.e. 30), $K=2$ performs better. $K=$ inf have no better performances in all the situations; it confirms that a bound on the generated states increase the exploration's speed. Moreover, we notice that exploration efficacy (ADRS and convergence speed) is not affected by $K$ since it strictly depends on $\lambda$, thus it is suggested to tune $\mathrm{K}$ such that the exploration time is minimized.

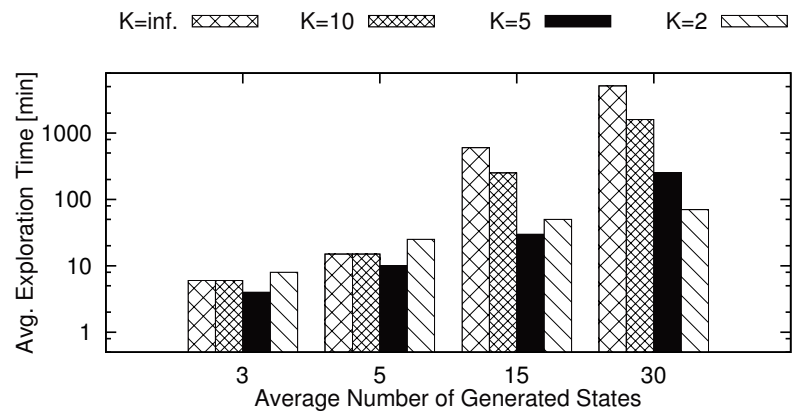

Figure 6: Amount of time (in minutes) required for the exploration with different values of $K$ ( $K=$ inf. refers to the original algorithm). The average number of generated states depends on the chosen $\lambda$ and movement vectors' accuracy 


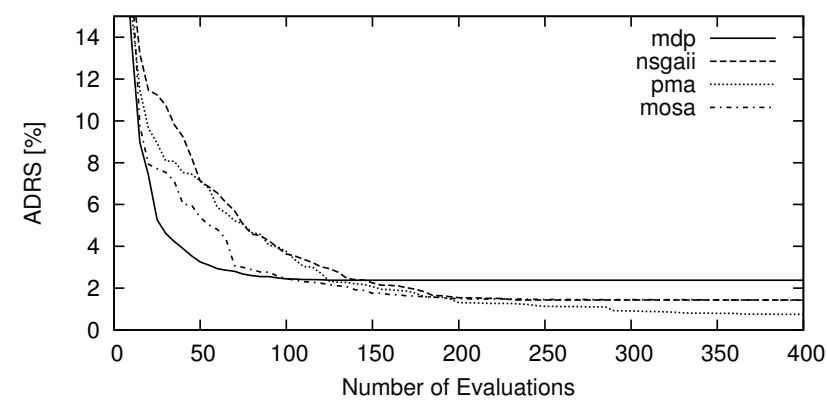

Figure 7: ADRS per Number of Evaluations in Scenario 1

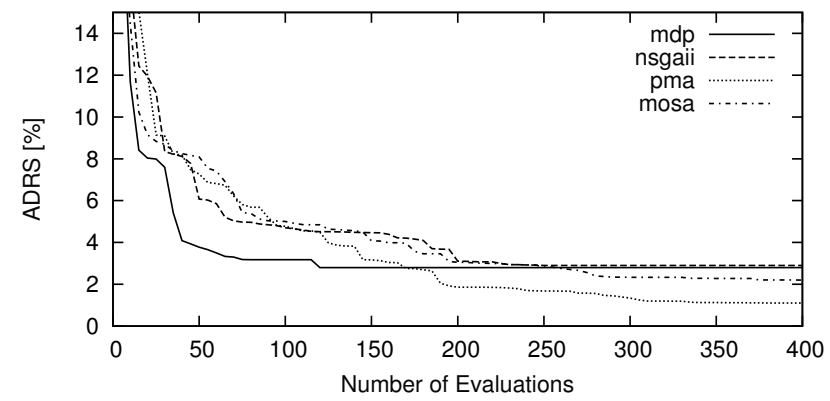

Figure 8: ADRS per Number of Evaluations in Scenario 2

The proposed algorithm is able to reduce/control the exponential growth of the number of parallel executions with respect to the original approach presented in [5] (where the threshold $\mathrm{K}$ has not been defined). Then, in addition to improving the quality of the final solution, the proposed approach is also able to reduce the number of explorations to be performed, thus reducing the computational costs and time required by the algorithm itself.

\subsection{ADRS and Number of Evaluations}

The second set of experiments compares the proposed MDP with three state-of-the-art multiobjective optimization algorithms: controlled non-dominated sorting genetic algorithm (NSGA-II), Pareto memetic algorithm (PMA) and multiple objective simulated annealing (MOSA). The MOMHLib ++ [1] library was used as a reference implementation of these algorithms. In order to ensure a fair comparison, all the algorithms exploits the technique described in Section 5.4 to generate the initial points.

Each configuration of all the optimization algorithms has been executed for 20 times in the four scenarios and the average ADRS has been evaluated. We compute the ADRS every 5 evaluations in order to understand its trend with respect to the number of evaluations. The results of these experiments are presented in Figure 7 (Scenario 1), Figure 8 (Scenario 2), Figure 9 (Scenario 3) and Figure 10 (Scenario 4), which show that MDP is able to reach a low ADRS (below 5\%) using less than 40 evaluations, while the other algorithms require from 100 to almost 300 evaluations to reach the same objective. This is a reduction of $60-87 \%$ in the number of required simulations. The time required to evaluate a single solution varies from 7 (Scenario 1 ) to 18 minutes (Scenario 4), that implies an exploration time of almost 5 hours for MDP and 12-36 hours for others in

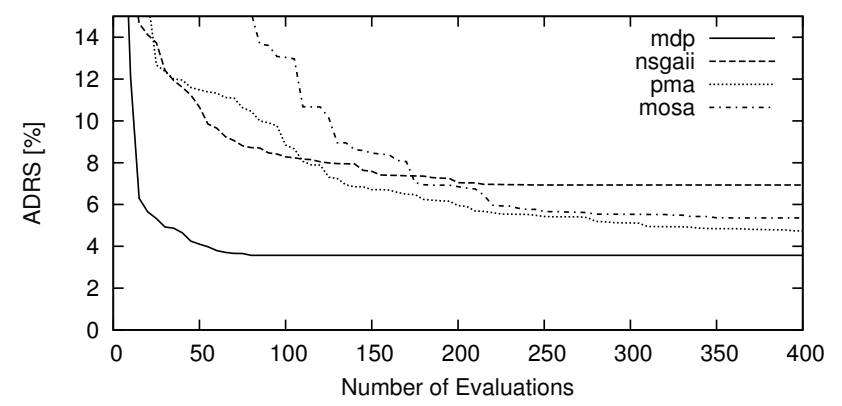

Figure 9: ADRS per Number of Evaluations in Scenario 3

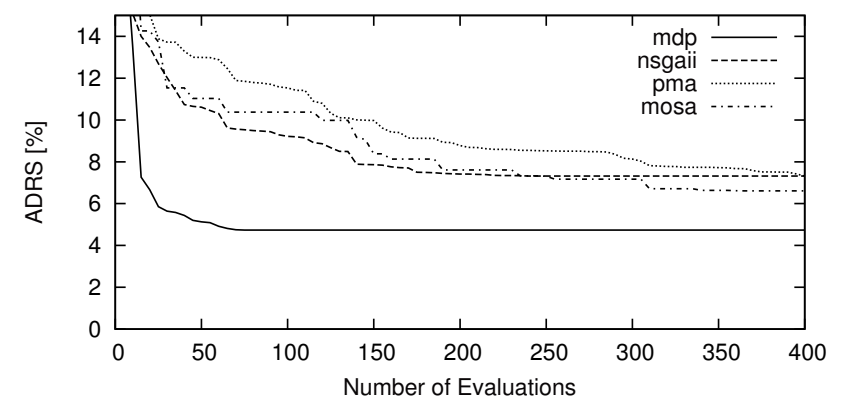

Figure 10: ADRS per Number of Evaluations in Scenario 4

Scenario 1, 12 hours for MDP and 30-60 hours for others in Scenario 4. These numbers illustrate the practical efficacy of the proposed approach for real-life WSN designs exploration and optimization.

As design space cardinality increases, the identification of the Pareto curve is more difficult, thus exploration efficiency decreases. It is interesting to notice that the reduction of effectiveness of MDP is considerably lower than the other algorithms (See Table 5). These results encourage the use of such algorithm on large design spaces.

During the Design Space Exploration, optimization algorithms should be run several times in order to guarantee the quality of the identified Pareto curve. Analyzing the standard deviation of the ADRS on the independent runs, we observed that standard deviation on MDP is the lowest. Table 6 summarized the computed standard deviations. A low standard deviation implies that the optimization algorithm requires few repeats to guarantee the quality; it

Table 5: Final ADRS of search Algorithms

\begin{tabular}{l|c|c|c|c}
\hline & MDP & NSGA-II & PMA & MOSA \\
\hline Scenario 1 & 2.34 & 1.42 & 0.83 & 1.43 \\
\hline Scenario 2 & 2.70 & 2.88 & 0.78 & 2.20 \\
\hline Scenario 3 & 3.52 & 6.52 & 4.76 & 5.36 \\
\hline Scenario 4 & 5.16 & 9.55 & 8.78 & 7.32 \\
\hline
\end{tabular}

Table 6: Standard Deviation of ADRS

\begin{tabular}{l|c|c|c|c}
\hline & MDP & NSGA-II & PMA & MOSA \\
\hline Scenario 1 & 0.34 & 1.20 & 1.01 & 1.34 \\
\hline Scenario 2 & 0.55 & 2.56 & 1.94 & 1.91 \\
\hline Scenario 3 & 0.64 & 2.44 & 2.38 & 2.81 \\
\hline Scenario 4 & 0.77 & 2.59 & 2.96 & 2.92 \\
\hline
\end{tabular}


further reduces the overall time required for Design Space Exploration.

\section{CONCLUDING REMARKS}

This paper has presented a technique to reduce the amount of simulations necessary to obtain the Pareto set of the design space exploration of Wireless Sensor Networks. The proposed technique uses models as soon as they provide an acceptable accuracy and simulates only when it is needed. The knowledge domain about slotted IEEE 802.15.4 has been extracted from the models of the state of the art. Experimental results have shown that proposed algorithm significantly improves the efficiency and scalability with respect to the classical algorithm. To confirm the efficacy of the technique, we also compared the proposed approach with semi-random algorithms such as NSGA-II, PMA and MOSA. Experimental results have shown that MDP reduces the number of simulations required to converge (ADRS lower than $5 \%$ ) from 60 to $87 \%$. This reduction is more effective as the cardinality of the design space increases, making it an effective approach for the design space exploration of WSNs.

\section{REFERENCES}

[1] MOMH: multiple objective meta heuristics [online]. available: http://home.gna.org/momh/.

[2] OMNET ++ castalia simulator, [Online] http://castalia.npc.nicta.com.au/.

[3] IEEE standard for information technology. 802.15.4 standard specification, 2006.

[4] L. S. Bai, R. P. Dick, P. H. Chou, and P. A. Dinda. Automated construction of fast and accurate system-level models for wireless sensor networks. In Design, Automation $\&$ Test in Europe Conference 8 Exhibition (DATE), 2011, pages 1-6. IEEE, Mar. 2011.

[5] G. Beltrame, L. Fossati, and D. Sciuto. Decision-Theoretic design space exploration of multiprocessor platforms. IEEE Transactions on Computer-Aided Design of Integrated Circuits and Systems, 29(7):1083-1095, July 2010.

[6] C. Buratti. A mathematical model for performance of IEEE 802.15.4 beacon-enabled mode. In Proceedings of the International Conference on Wireless Communications and Mobile Computing: Connecting the World Wirelessly, IWCMC '09, pages 1184-1190, New York, NY, USA, 2009. ACM.

[7] K. P. Ferentinos and T. A. Tsiligiridis. Adaptive design optimization of wireless sensor networks using genetic algorithms. Computer Networks, 51(4):1031-1051, Mar. 2007.

[8] O. Goussevskaia, R. Wattenhofer, M. M. Halldorsson, and E. Welzl. Capacity of arbitrary wireless networks. In IEEE INFOCOM 2009, pages 1872-1880. IEEE, Apr. 2009.

[9] J. He, Z. Tang, H. Chen, and Q. Zhang. An accurate and scalable analytical model for IEEE 802.15.4 slotted CSMA/CA networks. IEEE Transactions on Wireless Communications, 8(1):440-448, Jan. 2009.

[10] Z. He, J. Eggert, W. Cheng, X. Zhao, J. Millspaugh, R. Moll, J. Beringer, and J. Sartwell. Energy-aware portable video communication system design for wildlife activity monitoring. Circuits and Systems Magazine, IEEE, 8(2):25 -37, 2008.
[11] M. Kohvakka, M. Kuorilehto, M. Hännikäinen, and T. D. Hämäläinen. Performance analysis of IEEE 802.15.4 and ZigBee for large-scale wireless sensor network applications. In Performance evaluation of wireless ad hoc, sensor and ubiquitous networks, pages 48-57, New York, NY, USA, 2006. ACM.

[12] G. Lu, B. Krishnamachari, and C. S. Raghavendra. Performance evaluation of the IEEE 802.15.4 MAC for low-rate low-power wireless networks. In 2004 IEEE International Conference on Performance, Computing, and Communications, pages 701-706. IEEE, 2004.

[13] E. Meshkova, J. Riihijarvi, A. Achtzehn, and P. Mahonen. Exploring simulated annealing and graphical models for optimization in cognitive wireless networks. In IEEE Global Telecommunications Conference, 2009. GLOBECOM 2009, pages 1-8. IEEE, Dec. 2009.

[14] A. Munir and A. Gordon-Ross. An mdp-based application oriented optimal policy for wireless sensor networks. In Proceedings of the 7th IEEE/ACM international conference on Hardware/software codesign and system synthesis, CODES+ISSS '09, pages 183-192, New York, NY, USA, 2009. ACM.

[15] S. Nabar, J. Walling, and R. Poovendran. Minimizing energy consumption in body sensor networks via convex optimization. In Body Sensor Networks (BSN), Int. Conf. on, pages $62-67$, June 2010.

[16] M. Nabi, M. Blagojevic, T. Basten, M. Geilen, and T. Hendriks. Configuring multi-objective evolutionary algorithms for design-space exploration of wireless sensor networks. In Proceedings of the 4th ACM workshop on Performance monitoring and measurement of heterogeneous wireless and wired networks, PM2HW2N '09, pages 111-119, Tenerife, Canary Islands, Spain, 2009. ACM.

[17] G. Palermo, C. Silvano, and V. Zaccaria. ReSPIR: a response Surface-Based pareto iterative refinement for Application-Specific design space exploration. Computer-Aided Design of Integrated Circuits and Systems, IEEE Transactions on, 28(12):1816 -1829, Dec. 2009.

[18] P. Park, C. Fischione, and K. H. Johansson. Performance analysis of GTS allocation in beacon enabled IEEE 802.15.4. In 6th Annual IEEE Communications Society Conference on Sensor, Mesh and Ad Hoc Communications and Networks, 2009. SECON '09, pages 1-9. IEEE, June 2009.

[19] M. A. Pasha, S. Derrien, and O. Sentieys. System-Level synthesis for wireless sensor node controllers. ACM Transactions on Design Automation of Electronic Systems, 17(1):1-24, Jan. 2012.

[20] S. Russel and P. Norvig. Artificial Intelligence: Modern Approach. 1st ed. englewood cliffs, NJ: prentice hall edition, 1995.

[21] N. F. Timmons and W. G. Scanlon. Analysis of the performance of IEEE 802.15.4 for medical sensor body area networking. pages 16-24. IEEE, Oct. 2004.

[22] A. Varga. The OMNET++ discrete event simulation system. In Proceedings of the European Simulation Multiconference, pages 319-324. SCS - European Publishing House, 2001. 